\title{
Narcolepsy and the Pathological Aspects of Multiple Napping
}

\author{
Hartmut Schulz, Johanna Wilde-Frenz, Stephan Volk, \\ and Peter Geisler
}

\section{The Polyphasic-Monophasic Sleep-Wake Continuum}

The placement and duration of sleep within the nychthemeron is under the control of (1) homeostasis, (2) circadian rhythms, (3) individual demands and habits, and (4) environmental conditions. The first two factors are the basis for the two-process model of sleep (Borbély, 1982). Factor 3 was added by Webb (1988) as an essential behavioral component. Both this third factor and the fourth one are optional factors for sleep-wake regulation. According to the relative strength of each single factor and to their specific combination, very different temporal distributions of sleeping and waking can result.

Under a phylogenetic perspective, two strikingly different sleep-wake patterns can be discriminated, namely, a polyphasic one with many short sleep bouts in $24 \mathrm{hr}$, and a monophasic one with one long sleep episode. Biphasic (dawn/dusk) sleep-wake patterns are usually interpreted as a variant of the monophasic type. Humans and other primates such as apes and monkeys, display monophasic sleep-wake patterns with occasional naps (Tobler, 1989).

During ontogeny the sleep-wake pattern of humans develops from a polyphasic one in infancy, through a biphasic one with a regular nap in childhood, into the predominantly monophasic sleep-wake distribution of adulthood. It is mainly via the optional factors mentioned above that even in human adults the monophasic pattern can be transformed into a polyphasic one. Thus, for example, continuous bed rest is a condition that effectively dismantles the monophasic sleep-wake cycle (Campbell, 1984). But even if a destructuring of the monophasic sleep-wake cycle is allowed or explicitly demanded by the experimenter, subjects exhibit a bimodal 
distribution of sleep durations, which are either longer than $5 \mathrm{hr}$ (night sleep) or shorter than $4 \mathrm{hr}$ (nap sleep) with very little or no overlap between the two parts of the overall distribution (Zulley, 1988).

Although there are many occurrences of nap sleep in addition to night sleep in human adults (see Dinges and Broughton, 1989), we would like to stress the concept that the human adult basically exhibits a wellconsolidated monophasic sleep-wake cycle.

\section{The Sleep-Wake Distribution in Narcoleptic Patients}

In contrast to normal human subjects, narcoleptic patients suffer from a pronounced inability to maintain a monophasic sleep-wake cycle. These patients complain of having lost the ability to stay awake whenever they want to. The destruction of the monophasic sleep-wake cycle is due to three factors:

1. Excessive daytime sleepiness (EDS), which causes many long periods of reduced vigilance. At these times the patient has difficulties in concentrating, even if the situation demands a high degree of attention.

2. Overt episodes of daytime sleep, whereby the patient has no effective means to stay awake, even in situations when sleep is socially unacceptable or dangerous. In addition, situational factors like monotony, which may induce sleepiness even in normals, are especially deleterious for narcoleptic patients.

3. Night sleep disturbances with frequent sleep interruptions, some of them rather long. The great majority of patients complain of this symptom.

Table 16.1 shows standard sleep parameters that indicate the reduced sleep efficiency of narcoleptic patients in comparison to age-matched normal controls. The distribution of sleep and wakefulness within $24 \mathrm{hr}$ with sleep bouts during daytime and waking spells during nighttime suggests that the patients are caught in a vicious circle that prevents both effective sleeping and effective waking. To test this hypothesis, a single case experiment with daytime sleep deprivation was conducted.

\section{Daytime Sleep Deprivation in Narcolepsy}

A 42-yr-old, unemployed, male patient who suffered from EDS, sleep attacks, cataplexy, and hypnagogic hallucinations was studied for 60 consecutive days in the clinic. The core of the investigation was an extended episode (20 days) of daytime sleep deprivation. This episode was preceded by a 7-day wash-out phase subsequent to drug treatment with clomipramin 
TABLE 16.1. Comparison of night sleep data between 10 unmedicated narcoleptic patients and 10 age-matched normal subjects

\begin{tabular}{|c|c|c|}
\hline Sleep parameters & Patients & Controls \\
\hline TIB (min) & $473.7 \pm 17.6$ & $481.3 \pm 22.4$ \\
\hline TST (min) & $429.6 \pm 37.2$ & $457.3 \pm 15.1$ \\
\hline \multirow[t]{3}{*}{ SEI $(\%)$} & $90.6 \pm 5.6$ & $95.1 \pm 2.3$ \\
\hline & \multicolumn{2}{|c|}{ Latencies (min) } \\
\hline & Patients & Controls \\
\hline S1 & $4.4 \pm 4.4$ & $10.1 \pm 5.9$ \\
\hline S2 & $21.6 \pm 15.0$ & $13.9 \pm 7.0$ \\
\hline S3 & $38.8 \pm 14.7$ & $28.7 \pm 12.4$ \\
\hline S4 & $26.0 \pm 26.0$ & $45.0 \pm 74.8$ \\
\hline REM & $24.8 \pm 40.3$ & $78.9 \pm 19.2$ \\
\hline
\end{tabular}

\begin{tabular}{|c|c|c|c|c|c|c|}
\hline & \multicolumn{6}{|c|}{ Sleep stages (amounts) } \\
\hline & \multicolumn{2}{|c|}{ Minutes } & \multicolumn{2}{|c|}{$\%$ TIB } & \multicolumn{2}{|c|}{$\%$ TST } \\
\hline & Patients & Controls & Patients & Controls & Patients & Controls \\
\hline W & $44.1 \pm 25.7$ & $24.0 \pm 12.7$ & $9.4 \pm 5.6$ & $4.9 \pm 2.3$ & - & - \\
\hline S1 & $93.8 \pm 32.7$ & $28.0 \pm 13.3$ & $19.8 \pm 6.8$ & $5.8 \pm 2.8$ & $21.8 \pm 6.8$ & $6.1 \pm 2.9$ \\
\hline S2 & $202.6 \pm 42.9$ & $228.6 \pm 38.7$ & $42.8 \pm 8.9$ & $47.7 \pm 8.7$ & $47.3 \pm 9.5$ & $50.1 \pm 9.2$ \\
\hline S3 & $29.8 \pm 20.9$ & $42.1 \pm 17.2$ & $6.3 \pm 4.4$ & $8.8 \pm 3.6$ & $7.2 \pm 5.2$ & $9.2 \pm 3.7$ \\
\hline S4 & $19.7 \pm 30.4$ & $50.0 \pm 40.9$ & $4.1 \pm 6.2$ & $10.3 \pm 8.2$ & $4.3 \pm 6.5$ & $10.8 \pm 8.6$ \\
\hline REM & $79.2 \pm 17.1$ & $102.4 \pm 18.5$ & $16.7 \pm 3.4$ & $21.3 \pm 3.9$ & $18.4 \pm 3.2$ & $22.4 \pm 3.9$ \\
\hline MT & $4.4 \pm 3.4$ & $6.1 \pm 2.6$ & $0.9 \pm 0.7$ & $1.3 \pm 0.5$ & $1.0 \pm 0.8$ & $1.3 \pm 0.5$ \\
\hline Pause & $4.3 \pm 5.7$ & - & & - & & - \\
\hline
\end{tabular}

TIB = time in bed; TST = total sleep time; SEI = sleep efficiency index; $\mathrm{W}=$ time awake; $\mathrm{MT}=$ movement time.

(Anafranil ${ }^{\Phi}, 25 \mathrm{mg} /$ day) and followed by a 15 -day postintervention episode. Later the patient was first treated with the anticholinergic drug metixen (Tremarit ${ }^{\otimes}, 5-25 \mathrm{mg} /$ day), and thereafter with protryptilin (Maximed $^{\otimes}, 10 \mathrm{mg} /$ day). Here, only data from the day sleep-deprivation phase will be presented; the effects of the pharmacological treatments will be discussed elsewhere.

Scheduled bed rest with polygraphic recordings was between 2300 and 0700. During the daytime the patient was continuously observed by four accompanying persons taking turns who tried to prevent him from falling asleep by different activities, such as talking, playing, and walking outside. Sleepiness was rated every $15 \mathrm{~min}$ on visual analog scales by the patient as well as by one of the observers. There were many occasions where brief sleep attacks could not be avoided. In addition, the patient frequently appeared to be half-asleep and responded only slowly. As Table 16.2 indicates, self-reported sleep duration was less than 30 min during the whole 
TABLE 16.2. Daytime sleep deprivation in a 46-year-old narcoleptic male patient

\begin{tabular}{|c|c|c|c|c|c|}
\hline Days & Treatment & $\begin{array}{c}\text { Self-reported } \\
\text { duration of } \\
\text { daytime sleep (min) }\end{array}$ & $\begin{array}{l}\text { Sleep efficiency } \\
\text { index }(\%)\end{array}$ & 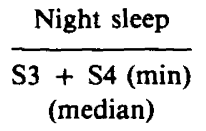 & $\begin{array}{c}\text { Subjective } \\
\text { sleep quality }{ }^{a}\end{array}$ \\
\hline $1-3$ & $\begin{array}{l}\text { Drug treatment } \\
\text { Clomipramin } \\
\text { (Anafranil } \\
25 \mathrm{mg} / \mathrm{d} \text { ) }\end{array}$ & - & $86 \pm 6$ & 54.3 & $6.5 \pm 0.1$ \\
\hline $4-10$ & $\begin{array}{l}\text { Drug withdrawal } \\
\text { wash-out }\end{array}$ & - & $79 \pm 5$ & 70.0 & $5.3 \pm 1.2$ \\
\hline $11-17$ & $\begin{array}{c}\text { Daytime sleep } \\
\text { deprivation }\end{array}$ & $<30$ & $87 \pm 5$ & & $3.2 \pm 1.5$ \\
\hline $18-24$ & $\begin{array}{c}\text { Daytime sleep } \\
\text { deprivation }\end{array}$ & $<30$ & $89 \pm 3$ & 78.5 & $3.0 \pm 0.8$ \\
\hline $25-30$ & $\begin{array}{c}\text { Daytime sleep } \\
\text { deprivation }\end{array}$ & $<30$ & $86 \pm 3$ & & $2.7 \pm 0.9$ \\
\hline $31-37$ & $\begin{array}{l}\text { Postinter- } \\
\text { vention (1st wk) }\end{array}$ & $115 \pm 46$ & $80 \pm 4$ & 74.0 & $4.3 \pm 1.8$ \\
\hline $38-45$ & $\begin{array}{l}\text { Postinter- } \\
\quad \text { vention (2nd wk) }\end{array}$ & $136 \pm 55$ & $82 \pm 2$ & & $3.4 \pm 0.8$ \\
\hline
\end{tabular}

${ }^{a} 0=$ very good; $10=$ very poor.

daytime sleep-deprivation period. This contrasted with the estimated mean day sleep times exceeding $100 \mathrm{~min}$ in all other parts of the study. Sleep efficiency was significantly higher during the daytime sleep-deprivation period than in the prior drug-withdrawal period or the subsequent postintervention period. These data suggest that the amount and continuity of night sleep are responsive to the amount of day sleep in narcoleptic patients.

Figure 16.1 displays the temporal distribution of REM sleep episodes for all 60 nights. Sleep onset REM episodes (SOREMs) were ubiquitous with the exception of the times with tricyclic medication. The anticholinergic drug metixen (nights 46-55) did not prevent the occurrence of SOREMs but obviously reduced the amount of REM sleep in the following $3 \mathrm{hr}$. This inhibition of REM sleep was followed by relatively high amounts of this sleep stage late in the nights.

From a clinical point of view, the treatment was not very effective, since (1) the patient showed many episodes of severe daytime sleepiness throughout the 3-wk deprivation period, and (2) there was no "transfer" of reduced daytime sleep or increased nighttime sleep efficiency into the postdeprivation period. Interestingly enough, none of the other symptoms of the patient (cataplexy, hypnagogic hallucinations) was either aggravated or improved by the sleep-deprivation procedure.

These results show that even an extended period with a strictly administered sleep-wake schedule was not sufficient to readapt a narcoleptic patient to a monophasic sleep-wake cycle. Nevertheless, the increased amount of 


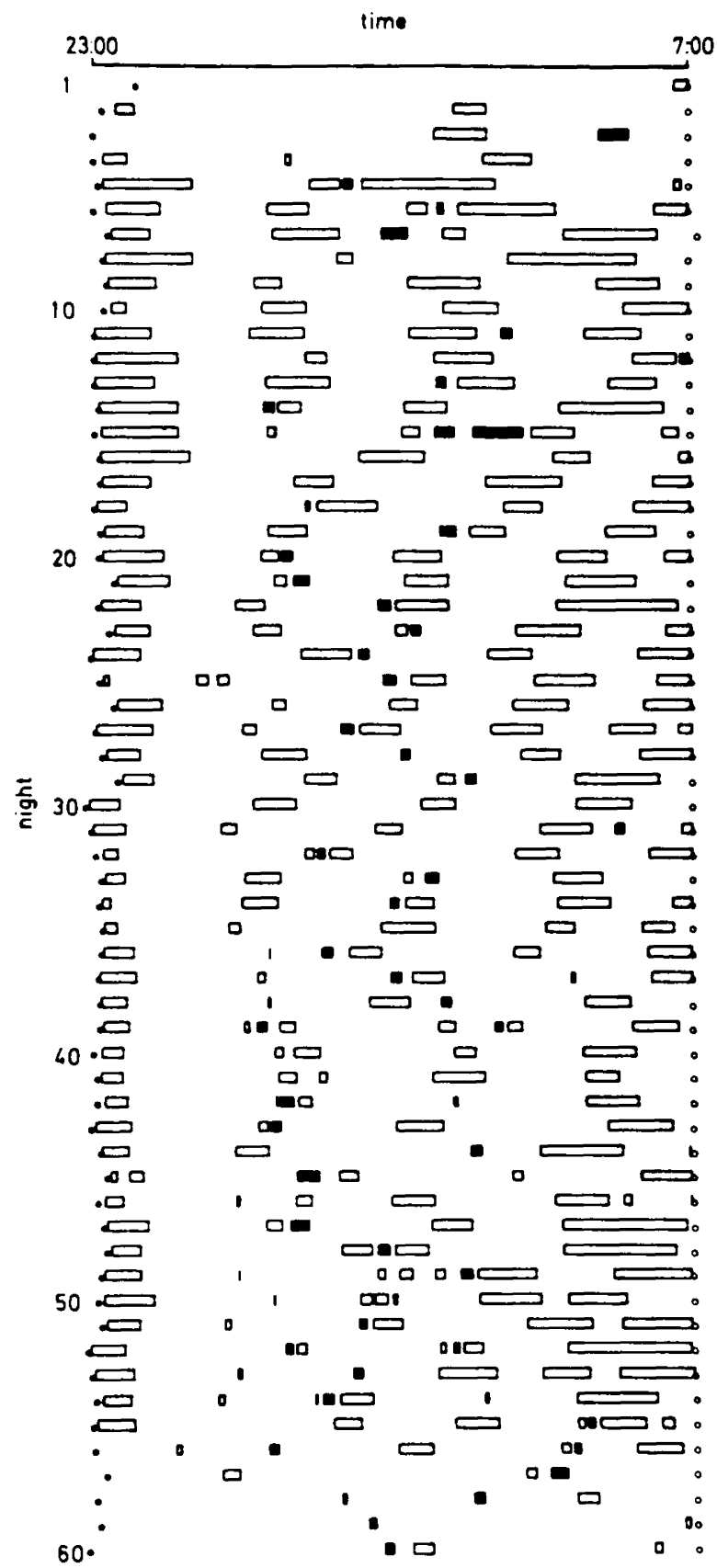

Figure 16.1. Distribution of REM sleep episodes during 60 consecutive night sleep recordings in a narcoleptic patient. For treatment conditions, see Table 16.2. Open bars represent REM sleep episodes, closed bars sleep interruptions (restroom visits). 
nighttime sleep after daytime sleep reduction indicates that factors that regulate sleep homeostasis are effective in narcolepsy. The same seems to be the case with the homeostatic regulation of slow wave sleep in narcolepsy (Volk et al., 1992).

If this is true, a weakness of other factors involved in sleep regulation, such as circadian processes, could be the basis of the pathophysiology of narcolepsy (cf. Kripke, 1976). To test the hypothesis that the circadian timing system is less effective in narcoleptic patients, we decided to study the sleep-wake behavior and other physiological variables of 2 patients under conditions of temporal isolation. Such experiments permit examination of the internal timing system after exclusion of all external time cues.

\section{The Effect of Temporal Isolation}

The first patient studied in a time-free environment (Andechs bunker facilities) was the same one who took part in the daytime sleep-deprivation study (see above). During the 3-wk observational period the patient showed a free-running rhythm of rectal temperature with a mean period of $24.7 \mathrm{hr}$. The sleep-wake distribution was rather similar to that under entrained conditions, with one major sleep episode within $24 \mathrm{hr}$ and multiple naps during the subjective day. From the beginning of temporal isolation the temperature rhythm desynchronized from the major sleep episodes, since the mean duration of the sleep-wake cycle $(32.9 \mathrm{hr})$ was substantially longer than the temperature rhythm (see Figure 16.2).

The second patient studied in temporal isolation was a 59-yr-old male patient with narcolepsy-cataplexy. Because of the severe form of the disease he was prematurely retired from work. Again, the sleep-wake cycle and other physiological variables were observed for $3 \mathrm{wk}$. While in the first week the alternation of wakefulness and the major sleep episodes was close to 24 $\mathrm{hr}$, in the last week the sleep-wake cycle was clearly shorter than $24 \mathrm{hr}$ and close to $21.5 \mathrm{hr}$ (see Figure 16.3).

Both cases studied in temporal isolation exhibited free-running sleepwake cycles with a clear separation of long sleep episodes (subjective night sleep) and shorter daytime sleep attacks. While in the first patient a desynchronization between the sleep-wake rhythm and the rhythm of body temperature could be established, such an analysis could not be performed for the second patient because his temperature curve was highly irregular due to masking effects.

More patients will have to be studied under temporal isolation to explore the coherence of the internal circadian timing system and its role in the disease process. A high frequency of sleep episodes during the subjective days in temporal isolation has also been reported for narcoleptic patients by Pollak et al. (1987). 


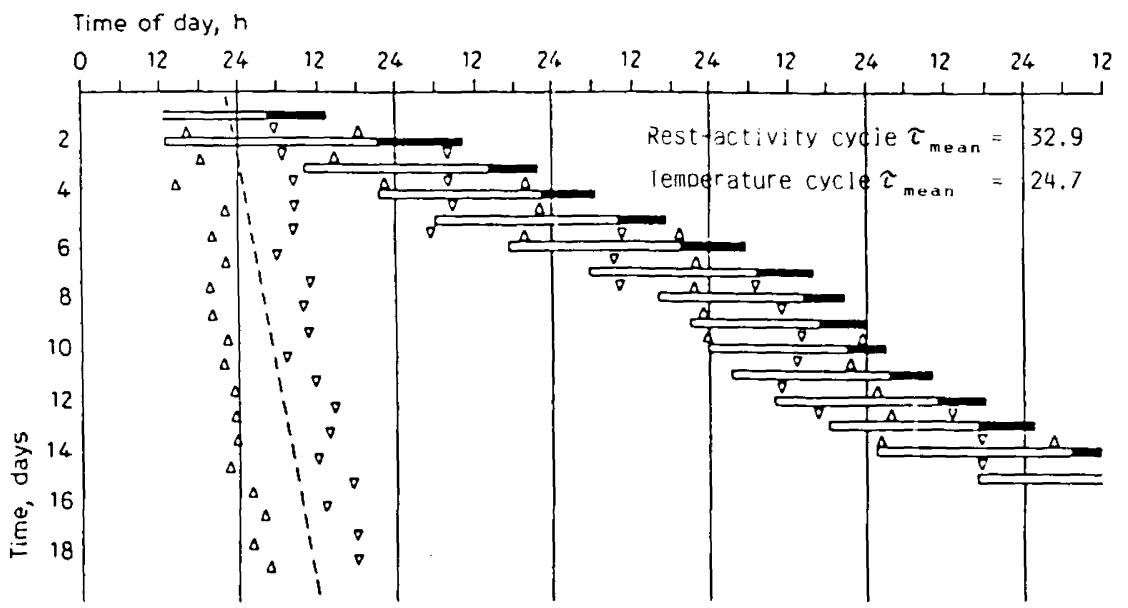

Figure 16.2. Free-running rhythms of sleep-wake and deep body temperature in a 42-yr-old male narcoleptic patient who lived in temporal isolation for $3 \mathrm{wk}$. Sleep is represented by the black part and wakefulness by the white part of the horizontal bars. The triangles pointing upward represent the circadian maxima of rectal body temperature, while those pointing downward represent the temperature minima.

\section{Performance Deficits in Narcoleptic Patients}

In this last section the deleterious effects of sleepiness and daytime sleep attacks on performance will be explored.

Although drops in vigilance and performance deficits are disabling consequences of the disease, few systematic studies on this topic have been published (Billiard, 1976; Valley and Broughton, 1983; Meier-Ewert, 1983; Levander and Sachs, 1985; Godbout and Montplaisir, 1986). Of these studies only one (Godbout and Montplaisir, 1986) tested performance, in this case forced-choice reaction time, at different times of the day. We used the critical flicker fusion (CFF) test to determine vigilance, because this test allows repeated measurements at short intervals. In addition, in our experience this test is sensitive enough to detect vigilance deficits in narcoleptic patients. The apparatus used (ZAK Ltd., Simbach/Inn) offers 50 stimuli per session, for $4 \mathrm{sec}$ each, in a randomized order. During each stimulus presentation four light dots are exposed to the subject simultaneously within a dark tube. One of the dots is the critical (flickering) stimulus. The other dots are steady. The task of the subject is to move a pointer next to the critical stimulus by turning a handbar within $4 \mathrm{sec}$. From the total of 50 runs the detection threshold (in $\mathrm{Hz}$ ) is computed.

Each patient or control subject was tested at 15 -min intervals for $10 \mathrm{hr}$ between 0800 and 1800 , equaling 40 test sessions. 


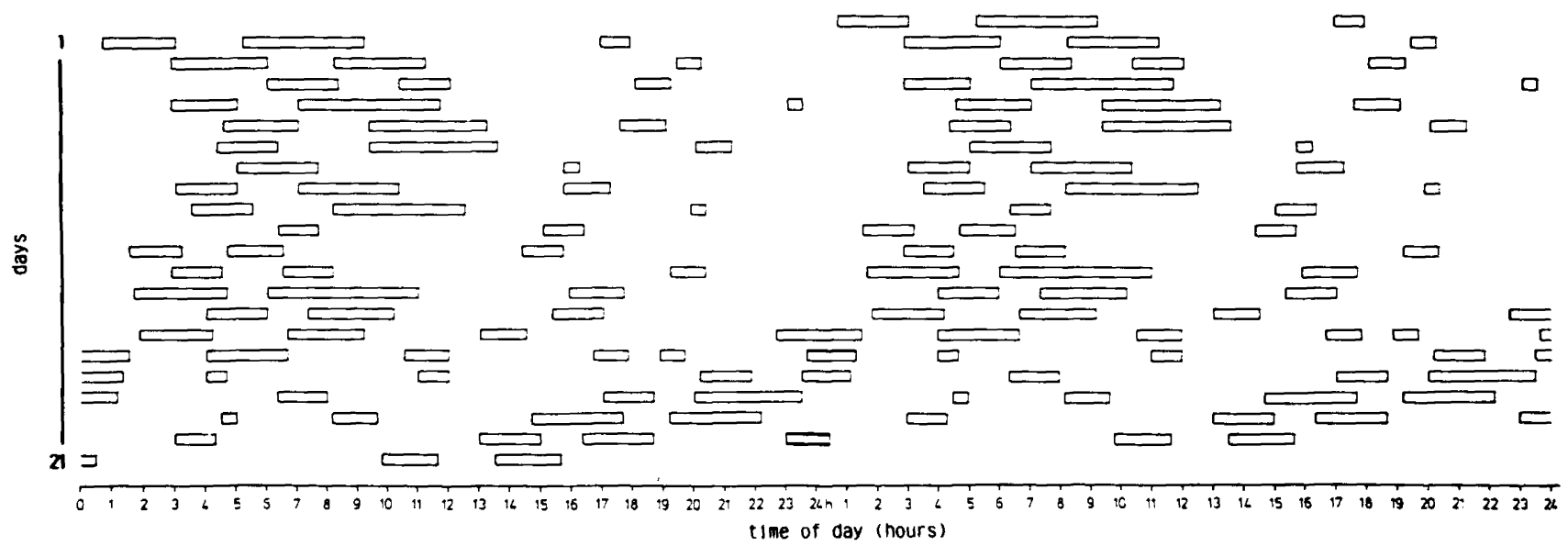

FIGURE 16.3. Double plot of the sleep episodes (subjective nights and naps) of a 59-yr-old male narcoleptic patient living in temporal isolation for $3 \mathrm{wk}$. Time of day (in hours) is given on the abscissa; days in the experiment are given on the ordinate. 
Ten unmedicated narcoleptic patients (4 males, 6 females, mean age: 42 yr, range: 31-58 yr) were compared with 10 age- and sex-matched normal control subjects (4 males, 6 females, mean age: $42 \mathrm{yr}$, range: $30-60 \mathrm{yrs}$ ).

Table 16.3 gives the mean performance data and the standard deviations for each of the 20 subjects. As can be seen, the mean performance rate of the group of narcoleptic patients is slightly lower than that of the control subjects $\bar{x}_{\mathrm{N}}=26.68$ vs $\left.\bar{x}_{\mathrm{C}}=28.61\right)$. This effect is mainly caused by the inability of the patients to perform on a stable level during the 10-hr test session. This is reflected in the significantly greater variances in the narcoleptic patients (see Figure 16.4). While all standard deviations in CFF performance were $<1$ in the control subjects, all standard deviations of the patients were $>1$, and the group mean was more than double that of the normal subjects.

Figure 16.5 shows three time courses of CFF performance, one from a normal subject and two from narcoleptic patients. While the majority of CFF values of the control subject fall into a small range $(27-28 \mathrm{~Hz})$, both patients show wide fluctuations. As indicated for both patients, the troughs in the performance curve occur at fairly regular intervals. The intervals between consecutive low performance values were 90 min (i.e., 6 measurement intervals) and 120 min (i.e., 8 measurement intervals), respectively, in these two cases. A detailed analysis of the data will be given elsewhere. However, it should be mentioned here that more patients displayed irregular than regular intervals between performance deficits.

These data demonstrate that the CFF test is a sensitive measure to detect fluctuations in cerebral vigilance in awake narcoleptic patients. Results from such a test could be validated against sleep propensity as measured by the Multiple Sleep Latency Test (MSLT).

TABLE 16.3. Critical flicker fusion thresholds (mean values and standard deviations) of 10 narcoleptic patients and 10 age-matched normal subjects

\begin{tabular}{lcllc}
\hline & \multicolumn{2}{c}{ Controls } \\
Subject & $\begin{array}{c}\text { CFF threshold } \\
\text { [mean } \pm \text { SD (min)] }\end{array}$ & & Subject & $\begin{array}{c}\text { CFF threshold } \\
\text { [mean } \pm \text { SD (min)] }\end{array}$ \\
\hline DIN & $23.6 \pm 3.5$ & MWK & $26.1 \pm 0.81$ \\
PEN & $22.7 \pm 1.8$ & MUK & $27.2 \pm 0.90$ \\
DON & $29.1 \pm 1.2$ & KUK & $27.0 \pm 0.58$ \\
SIN & $25.1 \pm 1.8$ & DOK & $29.0 \pm 0.66$ \\
WON & $25.8 \pm 2.5$ & JOK & $27.6 \pm 0.75$ \\
HIN & $25.7 \pm 2.4$ & LEK & $31.3 \pm 0.81$ \\
BKN & $28.9 \pm 1.2$ & RUK & $24.9 \pm 0.92$ \\
HAN & $23.3 \pm 2.8$ & GEK & $31.0 \pm 0.60$ \\
SCN & $27.9 \pm 1.6$ & POK & $30.4 \pm 0.55$ \\
HON & $31.8 \pm 2.8$ & HLK & $31.6 \pm 0.55$ \\
\hline
\end{tabular}




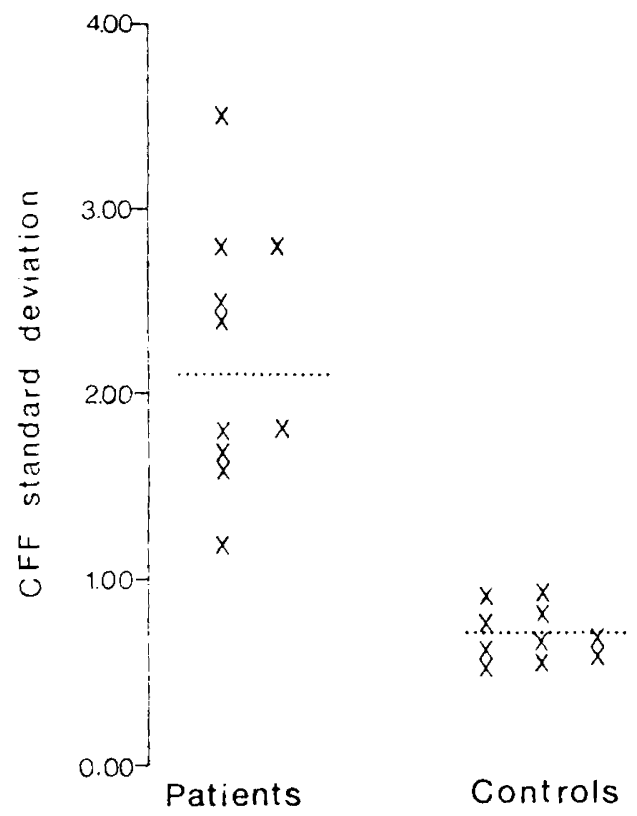

Figure 16.4. CFF standard deviations over 40 test sessions each for 10 narcoleptic patients and 10 matched normal control subjects.

\section{Conclusions}

Daytime sleep is a Janus-faced phenomenon. In the form of voluntarily placed naps it can be instrumental in functioning on a high level throughout the whole day. But if daytime sleep occurs involuntarily and is overwhelming, it has just the opposite effect. It then indicates, and may even contribute to, a state of insufficient functioning during the waking hours, as in different sleep-wake disturbances, especially narcolepsy.

In a series of diverse experiments some aspects of pathological sleepiness and napping in narcolepsy were studied. The flexibility and adaptability of the disorganized sleep-wake pattern to a strict monophasic rest-activity schedule was tested in a single case study by prolonged daytime sleep deprivation. Although sleep efficiency and the amount of slow wave sleep increased under this regimen, even 3 wks were not enough to transform the polyphasic sleep-wake pattern into a strictly monophasic one. This finding suggests that daytime sleep and nighttime wake episodes in narcolepsy may be the outcome of a permanent state border control disturbance (Broughton et al., 1986) rather than a sleep deficit or a sleep displacement.

This interpretation would also be in line with the results of the second set of experiments, where narcoleptic patients were observed under conditions of temporal isolation for $3 \mathrm{wk}$ each. Both patients clearly differentiated 

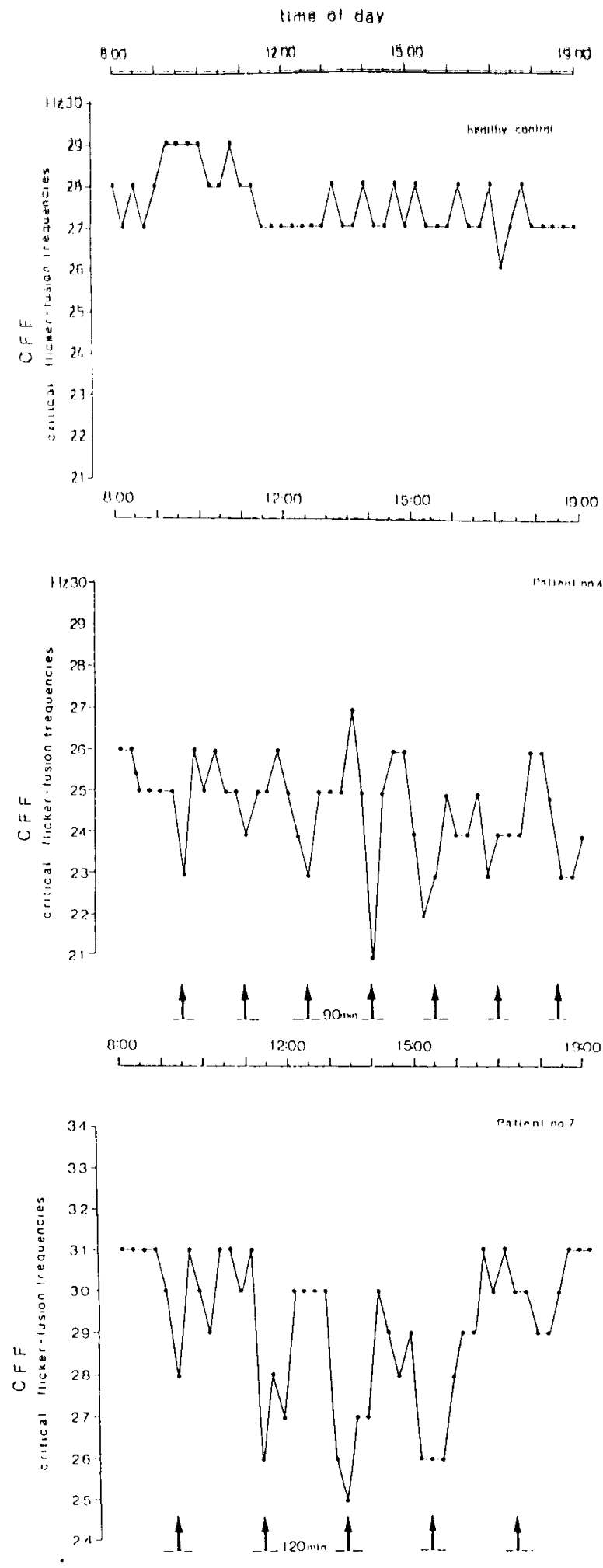
between subjective night sleep and nap sleep. This could be seen from the sleep-wake recordings and the diary entries. One interpretation would be that the strength of narcoleptics' internal circadian pacemakers is not much less than that of normal subjects. Otherwise one would have expected a much greater decay of the circadian component of the sleep-wake distribution in temporal isolation.

To make firm statements about the circadian periodicity of the body temperature and its phase relationship with the sleep-wake cycle, more patients will have to be studied. A detailed analysis of such data in narcoleptic patients is essential, since Zulley and Campbell (1985) have emphasized the role of naps for the concept of internal desynchronization.

Finally, the results of the CFF study show that the discrimination between sleeping and waking by electroencephalographic measures is not sufficient to represent the whole vigilance continuum. The method used here allowed the tracing of fluctuations of cerebral vigilance in narcoleptic patients, who for the most part would have been rated as awake according to polygraphic and behavioral criteria. It is of interest to note that some of the patients showed quite regular fluctuations of vigilance which fall into the range of Kleitman's Basic Rest-Activity Cycle (BRAC) theory (1982).

In the present context of polyphasic and ultrashort sleep-wake cycles this could mean that performance and EEG measures should be applied in combination to cover the whole vigilance continuum.

\section{References}

Billiard M (1976): Competition between the two types of sleep, and the recuperative function of REM versus NREM sleep in narcoleptics. In: Narcolepsy, Guilleminault C, Dement WC, Passouant P, eds. New York: Spectrum, pp 77-96

Borbély AA (1982): A two process model of sleep regulation. Hum Neurobiol 1:195-204

Broughton R, Valley V, Aguirre M, Roberts J, Suwalski E, Dunham W (1986): Excessive daytime sleepiness and the pathophysiology of narcolepsy-cataplexy. A laboratory perspective. Sleep 9:205-215

Campbell SS (1984): Duration and placement of sleep in a "disentrained" environment. Psychophysiology 21:106-113

Dinges DF, Broughton RJ, eds (1989): Sleep and Alertness: Chronobiological, Behavioral, and Medical Aspects of Napping. New York: Raven Press

Godbout R, Montplaisir J (1986): All-day performance variations in normal and narcoleptic subjects. Sleep 9:200-204

FIGURE 16.5. Three time courses of CFF performance over $10 \mathrm{hr}$-upper panel: data of a nomal subject; middle and lower panels: data of two nacoleptic patients. Optical fusion threshold $(\mathrm{Hz})$ is given on the ordinate. The arrows indicate equidistant rasters with 90-min intervals (middle panel) and 120-min intervals (lower panel). The rasters were consructed in such a way to give the best representation of local minima in the performance curves. 
Kleitman N (1982): Basic rest-activity cycle 22 years later. Sleep 5:311-317

Kripke DF (1976): Biological rhythm disturbances may cause narcolepsy. In: Narcolepsy, Guilleminault C, Dement WC, Passouant P, eds. New York: Spectrum, pp 475-483

Levander S, Sachs C (1985): Vigilance performance and autonomic function in narcolepsy: Effects of central stimulants. Psychophysiology 22:24-31

Meier-Ewert K (1983): Zur Begutachtung der Narkolepsien. Oeff Gesundheitswes 45:488-493

Pollak CP, Moline ML, Wagner DR (1987): Sleep times in narcoleptic subjects isolated from time cues. Sleep Res 16:406

Tobler I (1989): Napping and polyphasic sleep in mammals. In: Sleep and Alertness: Chronobiological, Behavioral, and Medical Aspects of Napping, Dinges DF, Broughton RJ, eds. New York: Raven Press, pp 9-30

Valley V, Broughton R (1983): The physiological (EEG) nature of drowsiness and its relation to performance deficits in narcoleptics. Electroencephalogr Clin Neurophysiol 55:243-251

Volk S, Schulz H, Yassouridis A, Wilde-Frenz J, Simon O (1992): The influence of two behavioral regimens on the distribution of sleep and wakefulness in narcoleptic patients. Sleep 13:136-142

Webb WB (1988): An objective behavioral model of sleep. Sleep 11:488-496

Zulley J (1988): The four-hour sleep wake cycle. Sleep Res 17:403

Zulley J, Campbell SS (1985): Napping behavior during "spontaneous internal desynchronization": Sleep remains in synchrony with body temperature. Hum Neurobiol 4:123-126 